\title{
Optimal counterfeiting attacks and generalizations for Wiesner's quantum money
}

\author{
Abel Molina, ${ }^{*}$ Thomas Vidick, ${ }^{+}$and John Watrous*
}

February 20, 2012

\begin{abstract}
We present an analysis of Wiesner's quantum money scheme, as well as some natural generalizations of it, based on semidefinite programming. For Wiesner's original scheme, it is determined that the optimal probability for a counterfeiter to create two copies of a bank note from one, where both copies pass the bank's test for validity, is $(3 / 4)^{n}$ for $n$ being the number of qubits used for each note. Generalizations in which other ensembles of states are substituted for the one considered by Wiesner are also discussed, including a scheme recently proposed by Pastawski, Yao, Jiang, Lukin, and Cirac, as well as schemes based on higher dimensional quantum systems. In addition, we introduce a variant of Wiesner's quantum money in which the verification protocol for bank notes involves only classical communication with the bank. We show that the optimal probability with which a counterfeiter can succeed in two independent verification attempts, given access to a single valid $n$-qubit bank note, is $(3 / 4+\sqrt{2} / 8)^{n}$. We also analyze extensions of this variant to higher-dimensional schemes.
\end{abstract}

\section{Introduction}

Wiesner's protocol for quantum money [Wie83] was a formative idea in quantum information processing. In this protocol, a bank generates a bank note composed of $n$ qubits: each qubit is initialized to a state chosen uniformly at random from the set $\{|0\rangle,|1\rangle,|+\rangle,|-\rangle\}$, and this choice of states is kept secret by the bank. The bank can later check the authenticity of a given note by performing a measurement on each of its qubits, in accordance with its secret record of their original states. (Each bank note is labeled with a unique serial number, so that all of the bank notes in circulation may be treated independently.) The security of Wiesner's scheme rests on the principle that quantum states cannot be cloned-that is, a malicious attacker, given access to a fixed supply of authentic bank notes, cannot generate a larger quantity of valid bank notes than those to which he was initially given access.

Although Wiesner's scheme was introduced almost three decades ago, to the best of our knowledge no rigorous analysis with explicit bounds on the security of the scheme exists in the literature. The intuition that the scheme's security follows from the no-cloning principle

\footnotetext{
*Institute for Quantum Computing and School of Computer Science, University of Waterloo. Supported by Canada's NSERC, CIFAR, and MITACS.

${ }^{\dagger}$ Computer Science and Artificial Intelligence Laboratory, Massachusetts Institute of Technology. Supported by the National Science Foundation under Grant No. 0844626.
} 
appears in [LSP98], and quantitatively one should be able to obtain exponential security guarantees from results such as proofs of the security of the BB84 quantum key exchange protocol [BB84, SP00, May01] or of uncloneable encryption [Got02]. In this paper we prove tight bounds on the security of Wiesner's quantum money scheme, through a simple and easily extended argument based on semidefinite programming.

We consider the specific situation in which a counterfeiter, given access to a single authentic bank note, attempts to create two bank notes having the same serial number that independently pass the bank's test for validity. We will call such attacks simple counterfeiting attacks. Our first main result is the following.

Theorem 1. The optimal simple counterfeiting attack against Wiesner's quantum money scheme has success probability exactly $(3 / 4)^{n}$, where $n$ is the number of qubits in each bank note.1]

Other types of attacks are not analyzed in this paper, but we must note their existence! For instance, a counterfeiter may attempt to create or copy bank notes through multiple interactions with the bank. One simple example of such an attack does not require counterfeiters to possess any bank notes to start with: by substituting one of two qubits of a Bell state for each qubit of a bank note, a counterfeiter can succeed in passing the bank's test for validity with probability $2^{-n}$, and then conditioned on having succeeded the counterfeiter will be guaranteed to hold a second valid bank note.2 One would therefore expect that the bank would charge a small fee for testing validity, or perhaps alert the authorities when an individual repeatedly makes failed attempts to validate bank notes, for otherwise counterfeiters have a very small but positive incentive to attack the protocol. Generally speaking, an analysis of attacks of this nature would seem to require a limit on the number of verification attempts permitted, or the specification of a utility function that weighs the potential gain from counterfeiting against the costs for multiple verifications. We expect that the semidefinite programming method used to prove Theorem 1 would be useful for analyzing such attacks, but we leave this as a problem for interested readers to consider.

We also consider simple counterfeiting strategies against quantum money schemes that generalize Wiesner's original scheme. These are the schemes obtained by varying the set of possible states that a quantum bank note may store, as well as the underlying probabilities for those states. We show that there is a scheme based on the repetition of a 4-state single-qubit scheme (i.e., having the same structure as Wiesner's) for which the optimal simple counterfeiting attack has success probability $(2 / 3)^{n}$, which is optimal among all schemes of that form. Furthermore, we show that any money scheme based on the use of $d$-dimensional bank notes is subject to a simple counterfeiting attack with success probability at least $2 /(d+1)$, and we describe a scheme for which this is the best one can do.

One drawback of Wiesner's money scheme is that, not only does it involve communicating with a centralized bank in order to establish the authenticity of a given bank note 3 but it also requires quantum communication: bills have to be sent to the bank for verification. Gavinsky [Gav11] recently introduced an alternative scheme in which bills can be authenticated using only classical communication with the bank.

\footnotetext{
${ }^{1}$ Wiesner [Wie83] in fact arrived at a similar bound, but through a not-so-rigorous argument!

${ }^{2}$ Lutomirski [Lut10] considered a related scenario where the bank kindly provides counterfeiters with access to a bank note's post-measurement qubits, regardless of whether validity was established. He proved that $O(n)$ verification attempts are sufficient to break the protocol in this setting.

${ }^{3}$ There has also been work in recent years on creating quantum money schemes that do not require any communication with the bank in order to verify a bank note, but this is only possible under computational assumptions $\left[\mathrm{FGH}^{+} 10, \mathrm{LAF}^{+} 10\right.$, Aar09].
} 
We consider the following procedure for classical verification of an $n$-qubit bank note, constructed as in Wiesner's scheme. The bank sends the user a random challenge $c \in\{0,1\}^{n}$. An honest user should measure the $i$-th qubit in the computational basis if $c_{i}=0$, or in the Hadamard basis if $c_{i}=1$, and send the measurement outcomes $b \in\{0,1\}^{n}$ to the bank. The bank validates the bank note if and only if whenever $c_{i}$ corresponded to the basis in which qubit $i$ was encoded, $b_{i}$ describes the correct outcome. (A similar scheme was independently introduced recently in $\left[\mathrm{PY}^{+}{ }^{+11} \mid\right.$.) In this setting, a simple counterfeiting attack is one in which a counterfeiter tries to succeed in two independent authentications with the bank, given access to a single valid bank note. Our second main result is the following.

Theorem 2. For the classical-verification analogue of Wiesner's quantum money scheme, the optimal simple counterfeiting attack has success probability exactly $(3 / 4+\sqrt{2} / 8)^{n}$, for $n$ being the number of qubits in each bank note.

As for Theorem 1, our proof of Theorem 2 follows from the use of semidefinite programming techniques. In addition we show that, contrary to the quantum-verification setting, Wiesner's scheme is optimal as long as one considers only qubits: either changing the bases used to encode each qubit or increasing the number of possible bases will not improve the scheme's security against simple counterfeiting attacks. We also consider a natural generalization of this scheme to bank notes made of $d$-dimensional qudits, and prove that the optimal simple counterfeiting attack against it has success probability exactly $(3 / 4+1 /(4 \sqrt{d}))^{n}$.

Related work. The no-cloning theorem [WZ82] states that there is no perfect quantum cloning machine. This impossibility result relies on two assumptions: that we are trying to clone all possible states (of a given dimension), and that we are trying to do so perfectly. Relaxing either or both assumptions opens the way for a fruitful exploration of the possibility of approximate cloning machines. Most work in this area focuses on obtaining universal cloners-required to work for all possible input states-but that may not be perfect.

To quantify the quality of a cloner one has to settle on a figure of merit. Two main figures have been considered: the minimum (or, alternately, the average) overlap between one of the two output clones with the input state, or the joint overlap of both output clones with a tensor product of the input state with itself 4 Bužek and Hillery [BH96] determined the optimal universal qubit cloner in the first case, while Werner [Wer98] solved the general problem with respect to the second figure of merit.

In the setting of quantum money, however, the first assumption is also relaxed: a counterfeiter only needs to be successful in cloning the specific states that are used to create the bank notes. Work in this direction includes that of Bruß et al. [BCDM00], who determined the optimal cloner for the states used in Wiesner's original money scheme, and for the first figure of merit discussed above. While in this work we consider the second figure of merit, which is the one appropriate to the context of quantum money, our results can easily be extended to the first.

We use a semidefinite programming formulation of the problem, in which one can numerically determine the success probability of an optimal cloner, given any desired possible set of input states and underlying distribution. The connection between cloning of quantum states and semidefinite programming was observed by Audenaert and De Moor [ADM02], and has been used in the study of cloning by other researchers. (See, for instance, the survey of Cerf and

\footnotetext{
${ }^{4}$ In both cases, the specific distance measure used can also be varied. For instance, the trace distance and the HilbertSchmidt distance on density matrices have been considered.
} 
Fiurášek [CF06].) The formulation that we use is closely related to one used in [MW11], and can also be seen as a special case of a semidefinite programming framework for more general quantum strategies developed in [GW07].

Recent work of Pastawski et al. [PY] $\left.{ }^{+} 11\right]$ contains an analysis of a 6-state variant of Wiesner's money scheme, obtaining a tight bound of $(2 / 3)^{n}$ on optimal simple counterfeiting attacks. In addition, they show that the scheme can be made error-tolerant-the bank will accept a bank note as long as say $99 \%$ of the qubit measurements are correct, allowing for the money state to be slightly perturbed and still undergo a successful authentication, 5 They also consider a classicalverification variant of the scheme that is similar to (but somewhat less efficient than) the one we propose, obtaining exponential security guarantees.

Other works consider more general counterfeiting attacks than we do, and develop techniques that may be useful to extend our own results. In particular, Aaronson and Christiano [AC12] reduce security against general $m \mapsto m+1$ cloners (given $m$ copies of a bank note, produce $m+1$ quantum states that will be simultaneously accepted by the bank's verification procedure) to security against simple counterfeiting attacks of the type we consider (attackers on their "minischemes"). Pastawski et al. [PYJ $\left.{ }^{+} 11\right]$ show that auxiliary access to the bank's verification procedure does not help, provided the only information returned by the bank is a single bit, indicating success or failure. Indeed, intuitively this situation may be reduced to one in which the cloner has no access to such a verification oracle simply by guessing: because most attempts in verification will result in failure (otherwise we would already have a successful cloner), the bits returned do not contain much information.

Organization of the paper. We start with some preliminaries on quantum information theory and semidefinite programming in Section 2 . Section 3 contains our results on Wiesner's quantum money scheme and generalizations, while Section 4 describes our results on schemes with classical verification procedure.

\section{Preliminaries}

We assume the reader is familiar with the basics of quantum information theory, and suggest Nielsen and Chuang [NC00] to those who are not. The purpose of this section is to summarize some of the notation and basic concepts we make use of, and to highlight a couple of concepts that may be less familiar to some readers. The lecture notes [Wat11] may be helpful to readers interested in further details on these topics.

\subsection{Basic notation, states, measurements and channels}

For any finite-dimensional complex Hilbert space $\mathcal{X}$ we write $\mathrm{L}(\mathcal{X})$ to denote the set of linear operators acting on $\mathcal{X}, \operatorname{Herm}(\mathcal{X})$ to denote the set of Hermitian operators acting on $\mathcal{X}, \operatorname{Pos}(\mathcal{X})$ to denote the set of positive semidefinite operators acting on $\mathcal{X}, \operatorname{Pd}(\mathcal{X})$ to denote the set of positive definite operators acting on $\mathcal{X}$, and $\mathrm{D}(\mathcal{X})$ to denote the set of density operators acting on $\mathcal{X}$. For Hermitian operators $A, B \in \operatorname{Herm}(\mathcal{X})$ the notations $A \geq B$ and $B \leq A$ indicate that $A-B$ is positive semidefinite, and the notations $A>B$ and $B<A$ indicate that $A-B$ is positive definite.

Given operators $A, B \in \mathrm{L}(\mathcal{X})$, one defines the inner product between $A$ and $B$ as $\langle A, B\rangle=$ $\operatorname{Tr}\left(A^{*} B\right)$. For Hermitian operators $A, B \in \operatorname{Herm}(\mathcal{X})$ it holds that $\langle A, B\rangle$ is a real number and

\footnotetext{
${ }^{5}$ Our analysis can also be extended to this setting; see Section 3.4 for more details.
} 
satisfies $\langle A, B\rangle=\langle B, A\rangle$. For every choice of finite-dimensional complex Hilbert spaces $\mathcal{X}$ and $\mathcal{Y}$, and for a given linear mapping of the form $\Phi: \mathrm{L}(\mathcal{X}) \rightarrow \mathrm{L}(\mathcal{Y})$, there is a unique mapping $\Phi^{*}: \mathrm{L}(\mathcal{Y}) \rightarrow \mathrm{L}(\mathcal{X})$ (known as the adjoint of $\left.\Phi\right)$ that satisfies $\langle Y, \Phi(X)\rangle=\left\langle\Phi^{*}(Y), X\right\rangle$ for all $X \in \mathrm{L}(\mathcal{X})$ and $Y \in \mathrm{L}(\mathcal{Y})$.

A register is a hypothetical device that stores quantum information. Associated with a register $\mathrm{X}$ is a finite-dimensional complex Hilbert space $\mathcal{X}$, and each quantum state of $\mathrm{X}$ is described by a density operator $\rho \in \mathrm{D}(\mathcal{X})$. Qubits are registers for which $\operatorname{dim}(\mathcal{X})=2$. A measurement of $\mathrm{X}$ is described by a set of positive semidefinite operators $\left\{P_{a}: a \in \Sigma\right\} \subset \operatorname{Pos}(\mathcal{X})$, indexed by a finite non-empty set of measurement outcomes $\Sigma$, and satisfying the constraint $\sum_{a \in \Sigma} P_{a}=\mathbb{1}_{\mathcal{X}}$ (the identity operator on $\mathcal{X}$ ). If such a measurement is performed on $\mathrm{X}$ while it is in the state $\rho$, each outcome $a \in \Sigma$ is obtained with probability $\left\langle P_{a}, \rho\right\rangle$. A quantum channel is a completely positive and trace-preserving linear mapping of the form $\Phi: \mathrm{L}(\mathcal{X}) \rightarrow \mathrm{L}(\mathcal{Y})$ that describes a hypothetical physical process that transforms each state $\rho$ of a register $X$ into the state $\Phi(\rho)$ of another register $\mathrm{Y}$. The identity channel that does nothing to a register $\mathrm{X}$ is denoted $\mathbb{1}_{\mathrm{L}(\mathcal{X})}$.

\subsection{Linear mappings on spaces of operators}

Suppose $\operatorname{dim}(\mathcal{X})=d$ and assume that a fixed orthonormal basis $\{|1\rangle, \ldots,|d\rangle\}$ of $\mathcal{X}$ has been selected. With respect to this basis, one defines the Choi-Jamiołkowski operator $J(\Phi) \in \mathrm{L}(\mathcal{Y} \otimes \mathcal{X})$ of a linear mapping $\Phi: \mathrm{L}(\mathcal{X}) \rightarrow \mathrm{L}(\mathcal{Y})$ as

$$
J(\Phi)=\sum_{1 \leq i, j \leq d} \Phi(|i\rangle\langle j|) \otimes|i\rangle\langle j| .
$$

The mapping $J$ is a linear bijection from the space of mappings of the form $\Phi: \mathrm{L}(\mathcal{X}) \rightarrow \mathrm{L}(\mathcal{Y})$ to $\mathrm{L}(\mathcal{Y} \otimes \mathcal{X})$. It is well-known that $\Phi$ is completely positive if and only if $J(\Phi) \in \operatorname{Pos}(\mathcal{Y} \otimes \mathcal{X})$, and that $\Phi$ is trace-preserving if and only if $\operatorname{Tr}_{\mathcal{Y}}(J(\Phi))=\mathbb{1}_{\mathcal{X}}$ [Cho75, Jam72]. It is also well-known, and easy to verify, that

$$
\langle\phi|\Phi(|\psi\rangle\langle\psi|)| \phi\rangle=\langle\phi \otimes \bar{\psi}|J(\Phi)| \phi \otimes \bar{\psi}\rangle
$$

for any choice of vectors $|\psi\rangle \in \mathcal{X}$ and $|\phi\rangle \in \mathcal{Y}$, with complex conjugation taken with respect to the standard basis.

\subsection{Semidefinite programming}

Semidefinite programming is a topic that has found several interesting applications within quantum computing and quantum information theory in recent years. Here, we provide just a brief summary of semidefinite programming that is focused on the narrow aspects of it that we use. More comprehensive discussions can be found in [VB96, Lov03, dK02, BV04], for instance.

A semidefinite program is a triple $(\Phi, A, B)$, where

1. $\Phi: \mathrm{L}(\mathcal{X}) \rightarrow \mathrm{L}(\mathcal{Y})$ is a Hermiticity-preserving linear mapping, and

2. $A \in \operatorname{Herm}(\mathcal{X})$ and $B \in \operatorname{Herm}(\mathcal{Y})$ are Hermitian operators,

for some choice of finite-dimensional complex Hilbert spaces $\mathcal{X}$ and $\mathcal{Y}$. We associate with the

\begin{tabular}{|c|c|c|c|}
\hline \multicolumn{2}{|c|}{ Primal problem } & \multicolumn{2}{|c|}{ Dual problem } \\
\hline maximize: & $\langle A, X\rangle$ & minimize: & $\langle B, Y\rangle$ \\
\hline subject to: & $\begin{array}{l}\Phi(X)=B \\
X \in \operatorname{Pos}(\mathcal{X})\end{array}$ & subject to: & $\begin{array}{l}\Phi^{*}(Y) \geq A \\
Y \in \operatorname{Herm}(\mathcal{Y})\end{array}$ \\
\hline
\end{tabular}
triple $(\Phi, A, B)$ two optimization problems, called the primal and dual problems, as follows: 
The optimal primal value of this semidefinite program is

$$
\alpha=\sup \{\langle A, X\rangle: X \in \operatorname{Pos}(\mathcal{X}), \Phi(X)=B\},
$$

and the optimal dual value is

$$
\beta=\inf \left\{\langle B, Y\rangle: Y \in \operatorname{Herm}(\mathcal{Y}), \Phi^{*}(Y) \geq A\right\} .
$$

(It is to be understood that the supremum over an empty set is $-\infty$ and the infimum over an empty set is $\infty$, so $\alpha$ and $\beta$ are well-defined values in $\mathbb{R} \cup\{-\infty, \infty\}$. In this paper, however, we will only consider semidefinite programs for which $\alpha$ and $\beta$ are finite.)

It always holds that $\alpha \leq \beta$, which is a fact known as weak duality. The condition $\alpha=\beta$, which is known as strong duality, does not hold for every semidefinite program, but there are simple conditions known under which it does hold. The following theorem provides one such condition (that has both a primal and dual form).

Theorem 3 (Slater's theorem for semidefinite programs). Let $(\Phi, A, B)$ be a semidefinite program and let $\alpha$ and $\beta$ be its optimal primal and dual values.

1. If $\beta$ is finite and there exists a positive definite operator $X \in \operatorname{Pd}(\mathcal{X})$ for which $\Phi(X)=B$, then $\alpha=\beta$ and there exists an operator $Y \in \operatorname{Herm}(\mathcal{Y})$ such that $\Phi^{*}(Y) \geq A$ and $\langle B, Y\rangle=\beta$.

2. If $\alpha$ is finite and there exists a Hermitian operator $Y \in \operatorname{Herm}(\mathcal{Y})$ for which $\Phi^{*}(Y)>A$, then $\alpha=\beta$ and there exists a positive semidefinite operator $X \in \operatorname{Pos}(\mathcal{X})$ such that $\Phi(X)=B$ and $\langle A, X\rangle=\alpha$.

In words, the first item of this theorem states that if the dual problem is feasible and the primal problem is strictly feasible, then strong duality holds and the optimal dual solution is achievable. The second item is similar, with the roles of the primal and dual problems reversed.

\section{Wiesner's quantum money and simple generalizations}

Wiesner's quantum money scheme, and straightforward generalizations of it, may be modeled in the following way. An ensemble of pure quantum states $\mathcal{E}=\left\{\left(p_{k},\left|\psi_{k}\right\rangle\right): k=1, \ldots, N\right\}$ is fixed, and assumed to be known to all (including any would-be counterfeiters). When preparing a bank note, the bank randomly selects a key $k \in\{1, \ldots, N\}$ with probability $p_{k}$. The bank note's quantum system is initialized to the state $\left|\psi_{k}\right\rangle$, and the note is labeled by a unique serial number. The bank records the serial number along with the secret key $k$.

When an individual wishes to verify a bank note, she brings it to the bank. The bank looks up the key $k$ and measures the note's quantum state with respect to the projective measurement $\{\Pi, \mathbb{1}-\Pi\}$, for $\Pi=\left|\psi_{k}\right\rangle\left\langle\psi_{k}\right|$. The measurement outcome associated with $\Pi$ causes the bank note to be declared valid, while the outcome associated with $\mathbb{1}-\Pi$ causes the bank note to be declared invalid.

A simple counterfeiting attack against a scheme of the form just described attempts to create two copies of a bank note from one, and is considered to be successful if both copies independently pass the bank's verification procedure. We take the original bank note's quantum state to be stored in a register $X$ having associated Hilbert space $\mathcal{X}$. The registers storing the quantum states corresponding to the two copies of the bank note produced by a would-be counterfeiter will be called $Y$ and $Z$. The Hilbert spaces $\mathcal{Y}$ and $\mathcal{Z}$ associated with these registers are taken to be isomorphic to $\mathcal{X}$, but will retain distinct names for the sake of our analysis. 
Mathematically speaking, a simple counterfeiting attack is described by a quantum channel $\Phi$ transforming $\mathrm{X}$ to $(\mathrm{Y}, \mathrm{Z})$, taking the state $\rho \in \mathrm{D}(\mathcal{X})$ to the state $\Phi(\rho) \in \mathrm{D}(\mathcal{Y} \otimes \mathcal{Z})$. In order to be physically realizable, at least in an idealized sense, the channel $\Phi$ must correspond to a completely positive and trace preserving linear mapping of the form $\Phi: \mathrm{L}(\mathcal{X}) \rightarrow \mathrm{L}(\mathcal{Y} \otimes \mathcal{Z})$. Conditioned on the bank having chosen the key $k$, the probability of success for an attack described by $\Phi$ is given by $\left\langle\psi_{k} \otimes \psi_{k}\left|\Phi\left(\left|\psi_{k}\right\rangle\left\langle\psi_{k}\right|\right)\right| \psi_{k} \otimes \psi_{k}\right\rangle$. Averaging over the possible choices of $k$, the overall success probability of a counterfeiting attack is

$$
\sum_{k=1}^{N} p_{k}\left\langle\psi_{k} \otimes \psi_{k}\left|\Phi\left(\left|\psi_{k}\right\rangle\left\langle\psi_{k}\right|\right)\right| \psi_{k} \otimes \psi_{k}\right\rangle .
$$

\subsection{An SDP formulation of simple counterfeiting attacks}

We now describe how the optimal success probability of a counterfeiting strategy, which is represented by the supremum of the probability (2) over all valid channels $\Phi: \mathrm{L}(\mathcal{X}) \rightarrow \mathrm{L}(\mathcal{Y} \otimes \mathcal{Z})$, may be represented by a semidefinite program. A similar semidefinite programming formulation may be found in [ADM02, CF06, MW11], for instance.

The formulation makes use of the Choi-Jamiołkowski representation $J(\Phi)$ of a given channel $\Phi$, as described in Section 2. Combining the characterization of all such representations that correspond to quantum channels given there together with (1) and the expression (2), it is not hard to see that the optimal success probability of any simple counterfeiting strategy is given by the following semidefinite program:

$$
\begin{aligned}
& \text { Primal problem } \\
& \text { maximize: }\langle Q, X\rangle \\
& \text { subject to: } \operatorname{Tr}_{\mathcal{Y} \otimes \mathcal{Z}}(X)=\mathbb{1}_{\mathcal{X}} \\
& X \in \operatorname{Pos}(\mathcal{Y} \otimes \mathcal{Z} \otimes \mathcal{X}) \\
& \text { Dual problem } \\
& \text { minimize: } \operatorname{Tr}(Y) \\
& \text { subject to: } \mathbb{1}_{\mathcal{Y} \otimes \mathcal{Z}} \otimes Y \geq Q \\
& Y \in \operatorname{Herm}(\mathcal{X})
\end{aligned}
$$

where

$$
Q=\sum_{k=1}^{N} p_{k}\left|\psi_{k} \otimes \psi_{k} \otimes \overline{\psi_{k}}\right\rangle\left\langle\psi_{k} \otimes \psi_{k} \otimes \overline{\psi_{k}}\right| .
$$

(The dual problem is obtained from the primal problem in a routine way, as described in Section 2.)

Because the primal and dual problems are both strictly feasible (as follows by taking $X$ and $Y$ to be appropriately chosen multiples of the identity, for example), it follows from Theorem 3 that the optimal values for the primal and dual problems are always equal, and are both achieved by feasible choices for $X$ and $Y$.

\subsection{Analysis of Wiesner's original scheme (single-qubit case)}

To analyze Wiesner's original quantum money scheme, we begin by considering the single-qubit (or $n=1$ ) case. The analysis of the scheme for arbitrary values of $n$ will follow from known results concerning product properties of semidefinite programs, as is described later in Section 3.4.

In the single-qubit case, Wiesner's quantum money scheme corresponds to the ensemble

$$
\mathcal{E}=\left\{\left(\frac{1}{4},|0\rangle\right),\left(\frac{1}{4},|1\rangle\right),\left(\frac{1}{4},|+\rangle\right),\left(\frac{1}{4},|-\rangle\right)\right\}
$$


which yields the operator

$$
Q=\frac{1}{4}(|000\rangle\langle 000|+| 111\rangle\langle 111|+|+++\rangle\langle+++|+|---\rangle\langle---|)
$$

in the semidefinite programming formulation described above. We claim that the optimal value of the semidefinite program in this case is equal to $3 / 4$. To prove this claim, it is sufficient to exhibit explicit primal and dual feasible solutions achieving the value $3 / 4$. For the primal problem, the value $3 / 4$ is obtained by the solution $X=J(\Phi)$, for $\Phi$ being the channel

$$
\Phi(\rho)=A_{0} \rho A_{0}^{*}+A_{1} \rho A_{1}^{*}
$$

where

$$
A_{0}=\frac{1}{\sqrt{12}}\left(\begin{array}{ll}
3 & 0 \\
0 & 1 \\
0 & 1 \\
1 & 0
\end{array}\right) \quad \text { and } \quad A_{1}=\frac{1}{\sqrt{12}}\left(\begin{array}{ll}
0 & 1 \\
1 & 0 \\
1 & 0 \\
0 & 3
\end{array}\right)
$$

For the dual problem, the value $3 / 4$ is obtained by the solution $Y=\frac{3}{8} \mathbb{1}_{\mathcal{X}}$, whose feasibility may be verified by computing $\|Q\|=3 / 8$.

\subsection{Optimal single-qubit schemes}

It is natural to ask if the security of Wiesner's original scheme can be improved through the selection of a different ensemble $\mathcal{E}$ in place of the one considered in the previous section. The answer is "yes," as follows from our analysis of Wiesner's original scheme together with the results of $\left[\mathrm{PYJ}^{+} 11\right]$, wherein the authors consider the ensemble

$$
\mathcal{E}=\left\{\left(\frac{1}{6},|0\rangle\right),\left(\frac{1}{6},|1\rangle\right),\left(\frac{1}{6},|+\rangle\right),\left(\frac{1}{6},|-\rangle\right),\left(\frac{1}{6}, \frac{|0\rangle+i|1\rangle}{\sqrt{2}}\right),\left(\frac{1}{6}, \frac{|0\rangle-i|1\rangle}{\sqrt{2}}\right)\right\} .
$$

The operator $Q$ that one obtains is given by

$$
Q=\frac{1}{\operatorname{rank}(\Pi)}\left(\mathbb{1}_{\mathrm{L}(\mathcal{Y})} \otimes \mathbb{1}_{\mathrm{L}(\mathcal{Z})} \otimes \mathrm{T}\right)(\Pi)
$$

for $\Pi$ being the projection onto the symmetric subspace of $\mathcal{Y} \otimes \mathcal{Z} \otimes \mathcal{X}$ and T being the transposition mapping with respect to the standard basis of $\mathcal{X}$.

The optimal value of the corresponding semidefinite program is $2 / 3$. Indeed, a primal feasible solution achieving the value $2 / 3$ is given by $X=J(\Phi)$ for $\Phi$ being the channel

$$
\Phi(\rho)=A_{0} \rho A_{0}^{*}+A_{1} \rho A_{1}^{*}
$$

where

$$
A_{0}=\frac{1}{\sqrt{6}}\left(\begin{array}{ll}
2 & 0 \\
0 & 1 \\
0 & 1 \\
0 & 0
\end{array}\right) \quad \text { and } \quad A_{1}=\frac{1}{\sqrt{6}}\left(\begin{array}{ll}
0 & 0 \\
1 & 0 \\
1 & 0 \\
0 & 2
\end{array}\right) .
$$

(This channel is the optimal qubit cloner of Bužek and Hillery [BH96]. ) A dual feasible solution achieving the bound $2 / 3$ is given by $Y=\frac{1}{3} \mathbb{1}_{\mathcal{X}}$ (with this solution's feasibility following from a calculation of $\|Q\|=1 / 3$ ). 
It is interesting to note that the same bound $2 / 3$ can be obtained by a four-state ensemble

$$
\mathcal{E}=\left\{\left(\frac{1}{4},\left|\tau_{1}\right\rangle\right),\left(\frac{1}{4},\left|\tau_{2}\right\rangle\right),\left(\frac{1}{4},\left|\tau_{3}\right\rangle\right),\left(\frac{1}{4},\left|\tau_{4}\right\rangle\right)\right\},
$$

where $\left\{\left|\tau_{1}\right\rangle, \ldots\left|\tau_{4}\right\rangle\right\}$ are any four states forming a single qubit SIC-POVM [RBKSC04]. The operator $Q$ corresponding to any such ensemble is identical to the one (3) from the six-state ensemble above, and therefore yields the same optimal value for the semidefinite program.

The schemes just mentioned are the best possible single qubit schemes. To see this, one may simply consider the performance of $\Phi$ (i.e., the Bužek-Hillery cloner), for which it follows by a direct calculation that

$$
\langle\psi \otimes \psi|\Phi(|\psi\rangle\langle\psi|)| \psi \otimes \psi\rangle=\frac{2}{3}
$$

for every state $|\psi\rangle$. This shows that the optimal primal value, and therefore the optimal counterfeiting probability, is always at least $2 / 3$.

\subsection{Parallel repetitions of generalized Wiesner schemes}

Wiesner's original scheme may be viewed as the $n$-fold parallel repetition of a scheme wherein the spaces $\mathcal{X}, \mathcal{Y}$, and $\mathcal{Z}$ each represent a single qubit, and where the initial state of each bank note is a state chosen uniformly from the set $\{|0\rangle,|1\rangle,|+\rangle,|-\rangle\}$. That is, the preparation and verification of each $n$-qubit bank note is, from the bank's perspective, equivalent to the independent preparation and verification of $n$ single-qubit bank notes; and a successful counterfeiting attack is equivalent to a successful counterfeiting attack against all $n$ of the single-qubit notes. The value of $n$ plays the role of a security parameter, given that it becomes increasingly hard to successfully counterfeit $n$ single-qubit bank notes in a row, without failure, as $n$ grows large.

Now, there is nothing that forces a counterfeiter to attempt to counterfeit an $n$-qubit bank note by treating each of its $n$ qubits independently. However, it is easily concluded from the semidefinite programming formulation above that a counterfeiter gains no advantage whatsoever by correlating multiple qubits during an attack. This, in fact, is true for arbitrary choices of the ensemble $\mathcal{E}$, as follows from a general result of Mittal and Szegedy [MS07] regarding product properties of some semidefinite programs. (In our case, this property follows from the fact that the operator $Q$ defining the objective function in the primal problem is always positive semidefinite.)

In greater detail, let us consider the $n$-fold repetition of a scheme, in which a single repetition of the scheme gives rise to a semidefinite program determined by $Q \in \operatorname{Pos}(\mathcal{Y} \otimes \mathcal{Z} \otimes \mathcal{X})$. Let us write $\mathcal{X}_{j}, \mathcal{Y}_{j}$, and $\mathcal{Z}_{j}$ to denote copies of the spaces $\mathcal{X}, \mathcal{Y}$, and $\mathcal{Z}$ that represent the $j$-th repetition of the scheme, for $j=1, \ldots, n$, and let us write $\mathcal{X}^{\otimes n}=\mathcal{X}_{1} \otimes \cdots \otimes \mathcal{X}_{n}, \mathcal{Y}^{\otimes n}=\mathcal{Y}_{1} \otimes \cdots \otimes \mathcal{Y}_{n}$, and $\mathcal{Z}^{\otimes n}=\mathcal{Z}_{1} \otimes \cdots \otimes \mathcal{Z}_{n}$. The semidefinite program that describes the optimal simple counterfeiting attack probability for the $n$-fold repetition is as follows:

$$
\begin{aligned}
& \text { Primal problem } \quad \text { Dual problem } \\
& \text { maximize: }\left\langle W_{\pi}\left(Q^{\otimes n}\right) W_{\pi}^{*}, X\right\rangle \\
& \text { minimize: } \operatorname{Tr}(Y) \\
& \text { subject to: } \operatorname{Tr}_{\mathcal{Y}}{ }^{\otimes n} \otimes \mathcal{Z}^{\otimes n}(X)=\mathbb{1}_{\mathcal{X}}{ }^{\otimes n} \\
& X \in \operatorname{Pos}\left(\mathcal{Y}^{\otimes n} \otimes \mathcal{Z}^{\otimes n} \otimes \mathcal{X}^{\otimes n}\right) \\
& \begin{aligned}
\text { subject to: } & \mathbb{1}_{\mathcal{Y} \otimes n \otimes \mathcal{Z}^{\otimes n}} \otimes Y \geq W_{\pi}\left(Q^{\otimes n}\right) W_{\pi}^{*} \\
& Y \in \operatorname{Herm}\left(\mathcal{X}^{\otimes n}\right)
\end{aligned}
\end{aligned}
$$

In this semidefinite program, $\mathcal{W}_{\pi}$ is a unitary operator representing a permutation of Hilbert spaces:

$$
\begin{aligned}
W_{\pi} \mid\left(y_{1} \otimes z_{1} \otimes x_{1}\right) \otimes \cdots \otimes & \left.\left(y_{n} \otimes z_{n} \otimes x_{n}\right)\right\rangle \\
& =\left|\left(y_{1} \otimes \cdots \otimes y_{n}\right) \otimes\left(z_{1} \otimes \cdots \otimes z_{n}\right) \otimes\left(x_{1} \otimes \cdots \otimes x_{n}\right)\right\rangle
\end{aligned}
$$


for all choices of $\left|x_{j}\right\rangle \in \mathcal{X}_{j},\left|y_{j}\right\rangle \in \mathcal{Y}_{j}$, and $\left|z_{j}\right\rangle \in \mathcal{Z}_{j}$, for $j=1, \ldots, n$.

If the optimal value of the semidefinite program is $\alpha$ in the single-repetition case, then the optimal value of the semidefinite program for the $n$-fold repetition case is necessarily $\alpha^{n}$. This may be proved by considering the primal and dual solutions $X=W_{\pi}\left(X_{1} \otimes \cdots \otimes X_{n}\right) W_{\pi}^{*}$ and $Y=Y_{1} \otimes \cdots \otimes Y_{n}$, for $X_{1}, \ldots, X_{n}$ being optimal primal solutions and $Y_{1}, \ldots, Y_{n}$ being optimal dual solutions for the single-repetition semidefinite program. The values obtained by these solutions are both $\alpha^{n}$. Primal feasibility of $X$ is straightforward, while dual feasibility of $Y$ follows from the fact that $A \geq B \geq 0$ implies $A^{\otimes n} \geq B^{\otimes n}$ for all positive semidefinite $A$ and $B$.

\subsection{Threshold results}

One may also consider noise-tolerant variants of Wiesner's scheme, as was done in [PYJ $\left.{ }^{+} 11\right]$. In the setting discussed in the previous subsection where $n$ repetitions of a particular scheme are performed, we may suppose that the bank's verification procedure declares a bank note valid whenever at least $t$ out of $n$ repetitions succeed, for some choice of $t<n$, as opposed to requiring that all $n$ repetitions succeed.

One might hope that a similar analysis to the one in the previous subsection will lead to an optimal counterfeiting probability of

$$
\sum_{t \leq j \leq n}\left(\begin{array}{l}
n \\
j
\end{array}\right) \alpha^{j}(1-\alpha)^{n-j}
$$

for such a scheme, for $\alpha$ being the optimal counterfeiting probability for a single repetition. This is the probability of successful counterfeiting when each repetition is attacked independently. In general, however, this bound may not be correct: the main result of [MW11] demonstrates a related setting in which an analogous bound does not hold, and explains the obstacle to obtaining such a bound in general. However, for some schemes, including Wiesner's original scheme and all of the other specific schemes (including the classical verification ones in Section 4.2) discussed in this paper, this bound will be correct. Letting $d=\operatorname{dim} \mathcal{X}$, the specific assumptions that we require to obtain the bound (4) are that

$$
\sum_{k=1}^{N} p_{k}\left|\psi_{k}\right\rangle\left\langle\psi_{k}\right|=\frac{1}{d} \mathbb{1},
$$

and that $Y=\frac{\alpha}{d} \mathbb{1}_{\mathcal{X}}$ is an optimal dual solution to the single-repetition semidefinite program (from which it follows $\left.\|Q\|=\frac{\alpha}{d}\right)$.

To prove that these requirements are sufficient, let us introduce the following notation. We will write $Q_{1}$ in place of $Q$ to denote the operator that specifies the semidefinite program representing a successful counterfeiting attack, and we will also define

$$
Q_{0}=\sum_{k=1}^{N} p_{k}\left(\mathbb{1}_{\mathcal{Y} \otimes \mathcal{Z}}-\left|\psi_{k} \otimes \psi_{k}\right\rangle\left\langle\psi_{k} \otimes \psi_{k}\right|\right) \otimes\left|\overline{\psi_{k}}\right\rangle\left\langle\overline{\psi_{k}}\right|,
$$

which has a complementary relationship to $Q_{1}$; it represents a failure to counterfeit in a given repetition. The semidefinite program describing the optimal counterfeiting probability for the $n$ fold repetition scheme, where successes in $t$ repetitions are required for a validation, is then as follows: 


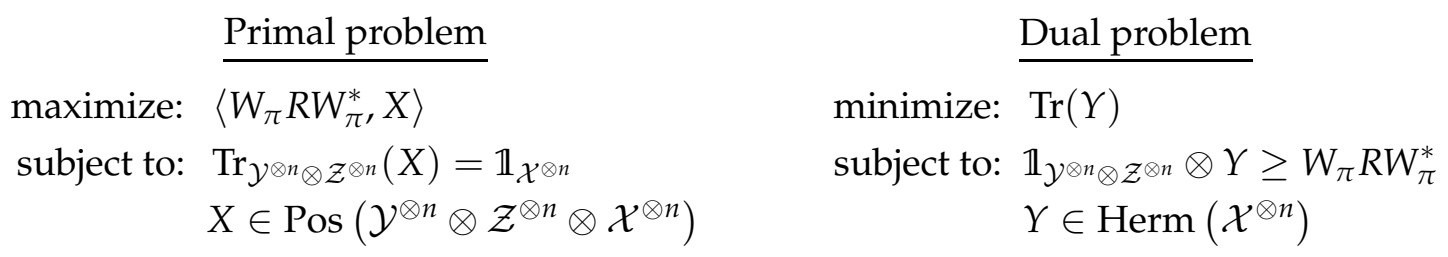

where

$$
R=\sum_{\substack{a_{1}, \ldots, a_{n} \in\{0,1\} \\ a_{1}+\cdots+a_{n} \geq t}} Q_{a_{1}} \otimes \cdots \otimes Q_{a_{n}} .
$$

To prove that the optimal value of this semidefinite program is given by the expression (4), it suffices to exhibit primal and dual feasible solutions achieving this value. As for the standard $n$-fold repetition case described in the previous subsection, it holds that $X=W_{\pi}\left(X_{1} \otimes \cdots X_{n}\right) W_{\pi}^{*}$ is a primal feasible solution that achieves the desired value, where again $X_{1}, \ldots, X_{n}$ are optimal primal solutions to the single-repetition semidefinite program. (This solution simply corresponds to an attacker operating independently and optimally in each repetition.) For the dual problem, we take

$$
Y=\|R\| \mathbb{1}_{\mathcal{X} \otimes n,}
$$

which is clearly dual-feasible. The condition (5) implies that $Q_{0}=\frac{1}{d} \mathbb{1}_{\mathcal{Y} \otimes \mathcal{Z} \otimes \mathcal{X}}-Q_{1}$, and a consideration of spectral decompositions of the commuting operators $Q_{0}$ and $Q_{1}$ reveals that

$$
\|R\|=\frac{1}{d^{n}} \sum_{t \leq j \leq n}\left(\begin{array}{l}
n \\
j
\end{array}\right) \alpha^{j}(1-\alpha)^{n-j},
$$

which establishes the required bound.

\subsection{Optimal schemes in higher dimensions}

We have observed that the best single-qubit variant of Wiesner's quantum money scheme has an optimal counterfeiting probability of $2 / 3$, and we know that the $n$-fold parallel repetition of this scheme has an optimal counterfeiting probability of $(2 / 3)^{n}$. Thus, bank notes storing a quantum state of dimension $d=2^{n}$ can have an optimal counterfeiting probability of $(2 / 3)^{n}$. It is natural to ask whether one can do better, using a scheme that is not given by the $n$-fold parallel repetition of a single qubit scheme.

The answer is that there are better schemes (provided $n>1$ ). More generally, for every $d$ representing the dimension of the state stored by a quantum bank note, there exist schemes whose optimal counterfeiting probability is equal to $2 /(d+1)$, which is the best that is possible: Werner's quantum cloning map [Wer98] will always succeed in counterfeiting any quantum bank note of dimension $d$ with probability $2 /(d+1)$. The following proposition shows that there exists a scheme that matches this bound in all dimensions $d$.

Proposition 4. Let $\mathcal{E}=\left\{p_{k},\left|\psi_{k}\right\rangle\right\}$ be any ensemble of d-dimensional states for which the operator

$$
Q=\sum_{k=1}^{N} p_{k}\left|\psi_{k} \otimes \psi_{k} \otimes \overline{\psi_{k}}\right\rangle\left\langle\psi_{k} \otimes \psi_{k} \otimes \overline{\psi_{k}}\right|
$$

is given by

$$
Q=\frac{1}{\operatorname{rank}(\Pi)}\left(\mathbb{1}_{\mathrm{L}\left(\mathbb{C}^{d}\right)} \otimes \mathbb{1}_{\mathrm{L}\left(\mathrm{C}^{d}\right)} \otimes \mathrm{T}\right)(\Pi)
$$


where $\mathrm{T}$ is the transposition mapping with respect to the standard basis of $\mathbb{C}^{d}$ and $\Pi$ is the orthogonal projector on the symmetric subspace of $\mathbb{C}^{d} \otimes \mathbb{C}^{d} \otimes \mathbb{C}^{d}$. Then no simple counterfeiting strategy can succeed against the money scheme derived from $\mathcal{E}$ with probability more than $2 /(d+1)$.

Before proving the proposition, we note that any ensemble $\mathcal{E}$ obtained from a complex projective (3,3)-design (also known as a quantum 3-design [AE07]) satisfies (6), and thus leads to an optimal $d$-dimensional money scheme. This also suggests that one might obtain more efficient schemes (i.e., involving less possible states for each part of the note) with security properties similar to the ones described here if approximate designs are considered instead.

Proof of Proposition 4 Because we are looking for an upper bound on the maximum counterfeiting probability, it suffices to construct a good feasible solution $Y$ to the dual SDP described in Section 3.1. We will choose $Y=\|Q\| \mathbb{1}_{\mathcal{X}}$, which is a feasible dual solution with corresponding objective value $\operatorname{Tr}(Y)=d\|Q\|$. We indicate how results from [EW01] may be used to show that $\|Q\|=2 /(d(d+1))$, proving the proposition.

The operator $Q$ commutes with all operators of the form $U \otimes U \otimes \bar{U}$, where $U$ is any unitary acting on $\mathbb{C}^{d}$. In Section VI.A of [EW01] it is shown that any such operator can be written as a linear combination of six conveniently chosen Hermitian operators $S_{+}, S_{-}, S_{0}, S_{1}, S_{2}, S_{3}$ (for a definition see Eqs. (25a)-(25f) of [EW01]). For our operator $Q$ we obtain the decomposition

$$
Q=\frac{1}{\operatorname{rank}(\Pi)}\left(\frac{1}{3} S_{+}+\frac{d+2}{6}\left(S_{0}+S_{1}\right)\right)
$$

where

$$
\begin{gathered}
S_{+}=\frac{\mathbb{1}+V}{2}-\frac{1}{2(d+1)}(X+X V+V X+V X V), \\
S_{0}+S_{1}=\frac{1}{d+1}(X+X V+V X+V X V),
\end{gathered}
$$

$V$ is the operator that permutes the first two registers on which $Q$ acts, and $X$ the partial transpose of the operator permuting the last two registers. Moreover, as shown in [EW01], $S_{+}$and $S_{0}$ are mutually orthogonal projections, $S_{0} S_{1}=S_{1} S_{0}=S_{1}, S_{+} S_{1}=S_{1} S_{+}=0$, and $S_{1}^{2}=S_{0}$. Hence, the decomposition (7) shows that the operator norm of $Q$ satisfies

$$
\|Q\|=\frac{1}{\operatorname{rank}(\Pi)} \frac{d+2}{3}=\frac{2}{d(d+1)}
$$

as $\operatorname{rank}(\Pi)=\left(\begin{array}{c}d+2 \\ 3\end{array}\right)$.

\section{Money schemes with classical verification}

In this section we introduce a natural variant of Wiesner's scheme, as well as higher-dimensional generalizations of it, in which the verification is done through classical communication with the bank. To distinguish the corresponding bank notes from the ones discussed in the previous section, we will call them tickets 6

\footnotetext{
${ }^{6}$ As we will see, successful verification of a ticket necessarily entails its destruction. This is unavoidable, as shown in [Gav11]. To avoid this issue one may concatenate many tickets together to create a single bill, that will be able to go through as many verification attempts as it contains tickets.
} 


\subsection{Description of quantum tickets}

A quantum ticket is defined in the same way as a bank note: it is a quantum state $\left|\psi_{k}\right\rangle$, where $k$ is a secret key kept by the bank, together with a unique serial number. We consider schemes in which the classical verification procedure has the following simple form. The user first identifies herself to the bank by announcing her ticket's serial number. The bank then sends her a classical "challenge" $c \in C$ chosen uniformly at random, where $C$ is some fixed finite set. Depending on $c$, an honest user will perform a measurement $\Pi_{c}=\left\{\Pi_{c}^{a}\right\}_{a \in A}$ on her ticket, and report the outcome $a$ to the bank. The bank then looks up the secret key $k$ associated with the user's ticket, and accepts $a$ if and only if the triple $(a, c, k)$ falls in a fixed, publicly known set $S$ of valid triples 7

A simple counterfeiting attack against such a scheme will attempt to use just one quantum ticket in order to successfully answer two independent challenges from the bank. Such a counterfeiter may be modeled by a collection of POVMs $A_{c_{1} c_{2}}=\left\{A_{c_{1} c_{2}}^{a_{1} a_{2}}\right\}_{a_{1} a_{2}}$, and its success probability is

$$
\sum_{k=1}^{N} p_{k} \frac{1}{|C|^{2}} \sum_{c_{1}, c_{2}} \sum_{\substack{\left(a_{1}, a_{2}\right): \\\left(a_{1}, c_{1}, k\right) \in S \\\left(a_{2}, c_{2}, k\right) \in S}}\left\langle\psi_{k}\left|A_{\mathcal{c}_{1} c_{2}}^{a_{1} a_{2}}\right| \psi_{k}\right\rangle,
$$

which is the "classical-verification" analogue of (2). By letting registers $Y$ and $Z$ contain the answers $a_{1}$ and $a_{2}$ respectively, and X contain the counterfeiter's input (the state $\left|\psi_{k}\right\rangle$ and the two challenges $c_{1}, c_{2}$ ), the problem of maximizing (8) over all possible counterfeiting strategies can be cast as a semidefinite program of the same form as the one introduced in Section 3.1, with the corresponding operator $Q$ defined as

$$
Q=\sum_{k=1}^{N} p_{k} \frac{1}{|C|^{2}} \sum_{c_{1}, c_{2}} \sum_{\substack{\left(a_{1}, a_{2}\right): \\\left(a_{1}, c_{1}, k\right) \in S \\\left(a_{2}, c_{2}, k\right) \in S}}\left|a_{1}\right\rangle\left|a_{2}\right\rangle\left|c_{1}, c_{2}, \psi_{k}\right\rangle\left\langle a_{1}\right|\left\langle a_{2}\right|\left\langle c_{1}, c_{2}, \psi_{k}\right| .
$$

Since $Q$ is diagonal on the first 4 registers, without loss of generality an optimal solution $X$ to the primal problem will be correspondingly block-diagonal,

$$
X=\sum_{a_{1}, a_{2}, c_{1}, c_{2}}\left|a_{1}, a_{2}, c_{1}, c_{2}\right\rangle\left\langle a_{1}, a_{2}, c_{1}, c_{2}\right| \otimes X_{c_{1} c_{2}}^{a_{1} a_{2}}
$$

and the SDP constraints are immediately seen to exactly enforce that $\left\{X_{c_{1} c_{2}}^{a_{1} a_{2}}\right\}_{a_{1} a_{2}}$ is a POVM for every $\left(c_{1}, c_{2}\right)$.

We note that the problem faced by the counterfeiter can be cast as a special instance of the more general state discrimination problem. Indeed, the counterfeiter's goal is to distinguish between the following: for every pair of possible answers $\left(a_{1}, a_{2}\right)$, there is a mixed state corresponding to the mixture over all states $\left|c_{1}\right\rangle\left|c_{2}\right\rangle\left|\Psi_{k}\right\rangle$ that for which $\left(a_{1}, a_{2}\right)$ would be a valid answer. (Each state is weighted proportionally to the probability of the pair $\left(c_{1}, c_{2}\right)$ of being chosen as challenges by the bank, and of $\left|\Psi_{k}\right\rangle$ being chosen as a bank note.) As such, the fact that the optimal counterfeiting strategy can be cast as a semidefinite program follows from similar formulations for the general state discrimination problem (as the ones considered in e.g. [EMV03]).

\footnotetext{
${ }^{7}$ For instance, the bank could accept all "plausible" answers, i.e., all $a$ such that $\left\langle\psi_{k}\left|\Pi_{c}^{a}\right| \psi_{k}\right\rangle>0$. This condition ensures that honest users are always accepted.
} 


\subsection{Analysis of a simple class of qudit schemes}

We further restrict our attention to a natural class of extensions of the classical-verification variant of Wiesner's scheme described in the introduction. The schemes we consider are parametrized by a dimension $d$ and two fixed bases $\left\{\left|e_{0}^{0}\right\rangle, \ldots,\left|e_{d-1}^{0}\right\rangle\right\}$ and $\left\{\left|e_{0}^{1}\right\rangle, \ldots,\left|e_{d-1}^{1}\right\rangle\right\}$ of $\mathbb{C}^{d} 8$ Each scheme is defined as the $n$-fold parallel repetition of a basic scheme in which $N=2 d$, the states $\left|\psi_{(t, b)}\right\rangle$ are the $\left|e_{t}^{b}\right\rangle$ for $t \in\{0, \ldots, d-1\}$ and $b \in\{0,1\}$, the random challenge is a bit $c \in\{0,1\}$, and the valid answers are $a=t$ if $b=c$, and any $a$ if $b \neq c$. Valid answers may be provided by an honest user who measures his ticket in the basis corresponding to $c$. By writing out the corresponding operator $Q$ and constructing a feasible solution to the dual SDP, we show the following lemma, from which Theorem 2 follows directly.

Lemma 5. For every simple counterfeiting attack against the n-qudit classical-verification scheme described above, the success probability is at most $\left(\frac{3}{4}+\frac{\sqrt{c}}{4}\right)^{n}$, where $c=\max _{s, t}\left|\left\langle e_{s}^{0} \mid e_{t}^{1}\right\rangle\right|^{2}$ is the effective overlap 9 If $d=2$, there is always a counterfeiting strategy that achieves this bound.

Proof. We first analyze simple counterfeiting attacks against the basic single-qudit scheme. Note that if both challenges from the bank are identical, the counterfeiter can answer both correctly with probability 1 by making the appropriate measurement on his qubit.

By symmetry, it suffices to consider the case where the first challenge is $c_{1}=0$ and the second is $c_{2}=1$. In this case the operator $Q$ becomes

$$
Q=\frac{1}{2 d} \sum_{s, t=0}^{d-1}|s\rangle\left\langle\left. s\right|_{\mathcal{Y}} \otimes \mid t\right\rangle\left\langle\left. t\right|_{\mathcal{Z}} \otimes\left(\left|e_{s}^{0}\right\rangle\left\langle\left. e_{s}^{0}\right|_{\mathcal{X}}+\mid e_{t}^{1}\right\rangle\left\langle e_{t}^{1}\right| \mathcal{X}\right) .\right.
$$

For $s, t \in\{0, \ldots, d-1\}$, let $V_{s, t}=\left|e_{s}^{0}\right\rangle\left\langle\left. e_{s}^{0}\right|_{\mathcal{X}}+\mid e_{t}^{1}\right\rangle\left\langle\left. e_{t}^{1}\right|_{\mathcal{X}}\right.$. As $Q$ is block-diagonal, the dual SDP is

$$
\begin{array}{ll}
\text { minimize: } & \operatorname{Tr}(Y) \\
\text { subject to: } & Y \geq \frac{1}{2 d} V_{s, t} \quad(\text { for all } s, t) \\
& Y \in \operatorname{Herm}\left(\mathbb{C}^{d}\right) .
\end{array}
$$

$V_{s, t}$ is a rank-2 Hermitian matrix whose eigenvalues are $1 \pm\left|\left\langle e_{s}^{0} \mid e_{t}^{1}\right\rangle\right|$. Hence, $Y=\frac{1+\sqrt{c}}{2 d} \mathbb{1}$ is a feasible solution to the dual problem with objective value $(1+\sqrt{c}) / 2$, leading to an upper bound on the best counterfeiting strategy with overall success probability at most $3 / 4+\sqrt{c} / 4$.

To finish the proof of the upper bound it suffices to note that the SDP has the same parallel repetition property as was described in Section 3.4 .

Finally, we show the "moreover" part of the claim. Relabeling the vectors if necessary, assume $\left|\left\langle e_{0}^{0} \mid e_{0}^{1}\right\rangle\right|=\sqrt{c}$. Let $\left|u_{0}\right\rangle$ be the eigenvector of $V_{0,0}$ with largest eigenvalue $1+\sqrt{c}$, and $\left|u_{1}\right\rangle$ the eigenvector with smallest eigenvalue. Using the observation that $\left|\left\langle e_{1}^{0} \mid e_{1}^{1}\right\rangle\right|=\sqrt{c}$, it may be checked that

$$
X=|0,0\rangle\left\langle 0,0|\otimes| u_{0}\right\rangle\left\langle u_{0}|+| 1,1\right\rangle\left\langle 1,1|\otimes| u_{1}\right\rangle\left\langle u_{1}\right|
$$

is a feasible solution to the primal SDP corresponding to (9) (as expressed in Section 3.1) with objective value $(1+\sqrt{c}) / 2$, proving that the optimum of (9) is exactly $(1+\sqrt{c}) / 2$.

\footnotetext{
${ }^{8}$ It is easy to see that increasing the number of bases will only result in weaker security: indeed, the more the bases the less likely it is that the bank's randomly chosen challenge will match the basis used to encode each qudit.

${ }^{9}$ For any two bases of $\mathbb{C}^{d}, c \geq 1 / d$, and this is achieved for a pair of mutually unbiased bases. This quantity also arises naturally in the study of uncertainty relations (see e.g. [TR11]), of which our result may be seen as giving a special form.
} 


\subsection{A matching lower bound}

Let $d$ be a fixed dimension. We introduce a quantum ticket scheme for which the upper bound derived in the previous section is tight. For $d=2$ our scheme recovers the one that is derived from Wiesner's original quantum money. Let $X_{d}$ and $Z_{d}$ be the generalized Pauli matrices, acting as

$$
X_{d}:|i\rangle \rightarrow|i+1 \quad \bmod d\rangle \quad \text { and } \quad Z_{d}:|i\rangle \rightarrow \omega^{i}|i\rangle,
$$

where $\omega=e^{2 i \pi / d}$. Let $F$ be the quantum Fourier transform over $\mathbb{Z}_{d}$,

$$
F:|i\rangle \rightarrow \frac{1}{\sqrt{d}} \sum_{j} \omega^{i j}|j\rangle,
$$

and note that $X_{d}=F Z_{d} F^{\dagger}$. Let $\left\{\left|e_{t}^{0}\right\rangle\right\}$ be the basis defined by $\left|e_{t}^{0}\right\rangle=\left(X_{d}\right)^{t}|0\rangle=|t\rangle$, and $\left\{\left|e_{t}^{1}\right\rangle\right\}$ the Fourier-transformed basis $\left|e_{t}^{1}\right\rangle=F\left|e_{t}^{0}\right\rangle=\left(Z_{d}\right)^{t} F|0\rangle$ for every $t$. Then

$$
\left|\left\langle e_{s}^{0} \mid e_{t}^{1}\right\rangle\right|=|\langle s|F| t\rangle|=\frac{1}{\sqrt{d}}
$$

for every $s, t$ : the corresponding overlap is $c=1 / d$. Lemma 5 shows that the optimal cloner achieves success at most $3 / 4+1 /(4 \sqrt{d})$. The following lemma states a matching lower bound.

Lemma 6. There is a cloner for the n-qudit ticket scheme described above which successfully answers both challenges with success probability $\left(\frac{3}{4}+\frac{1}{4 \sqrt{d}}\right)^{n}$.

Proof. We describe a cloner that acts independently on each qudit, succeeding with probability $\frac{3}{4}+\frac{1}{4 \sqrt{d}}$ on each qudit 10 Let

$$
|\psi\rangle=(2+2 / \sqrt{d})^{-1 / 2}(|0\rangle+F|0\rangle),
$$

and for every $(s, t)$ let $P_{s, t}$ be the rank 1 projector on the unit vector $X_{d}^{s} Z_{d}^{t}|\psi\rangle$. As a consequence of Schur's lemma, $\sum_{s, t} \frac{1}{d} P_{s, t}=\mathbb{1}$, so that $\left\{P_{s, t} / d\right\}$ is a POVM.

The cloner proceeds as follows: if the challenge is either 00 or 11, he measures in the corresponding basis and sends the resulting outcome as answer to both challenges. In this case he is always correct. In case the challenge is either 01 or 10, he measures the ticket using the POVM $\left\{P_{s, t} / d\right\}$, and uses $s$ as answer to the challenge " 0 " and $t$ as answer to the challenge " 1 ". Because the two challenges are distinct, only one of them corresponds to the actual basis in which the ticket was encoded. Without loss of generality assume this is the " 0 " basis, so that the ticket is $\left|e_{s}^{0}\right\rangle=|s\rangle$. The probability that the cloner obtains the correct outcome $s$ is

$$
\begin{aligned}
\frac{1}{d} \sum_{t} \operatorname{Tr}\left(P_{s, t}|s\rangle\langle s|\right) & =\frac{1}{d} \sum_{t}\left|\left\langle s\left|X_{d}^{s} Z_{d}^{t}\right| \psi\right\rangle\right|^{2} \\
& =\frac{1}{d} \sum_{t}\left|\left\langle 0\left|Z_{d}^{t}\right| \psi\right\rangle\right|^{2} \\
& =|\langle 0 \mid \psi\rangle|^{2},
\end{aligned}
$$

because, for every $t$, it holds that $\langle 0| Z_{d}^{t}=\omega^{t}\langle 0|$. To conclude, it suffices to compute

$$
|\langle 0 \mid \psi\rangle|^{2}=\frac{1}{2+2 / \sqrt{d}}|\langle 0 \mid 0\rangle+\langle 0|F| 0\rangle|^{2}=\frac{1}{2}\left(1+\frac{1}{\sqrt{d}}\right) .
$$

\footnotetext{
${ }^{10}$ The analysis is very similar to one that was done in [VW11], in a different context but for essentially the same problem.
} 
Acknowledgments We thank Scott Aaronson for his question 11 on Theoretical Physics Stack Exchange that originated the results in this paper as an answer, and Peter Shor for pointing out the connection between the channel representing an optimal attack on Wiesner's quantum money, and the optimal cloners studied in [BCDM00] and [BH96]. JW thanks Debbie Leung and Joseph Emerson for helpful discussions. AM thanks Michael Grant and Stephen Boyd for their creation of the CVX software.

\section{References}

[Aar09] S. Aaronson. Quantum copy-protection and quantum money. In Proceedings of the 24th Annual IEEE Conference on Computational Complexity, pages 229-242, 2009.

[AC12] S. Aaronson and P. Christiano. Quantum money from hidden subspaces. In preparation, 2012.

[ADM02] K. Audenaert and B. De Moor. Optimizing completely positive maps using semidefinite programming. Physical Review A, 65:030302, 2002.

[AE07] A. Ambainis and J. Emerson. Quantum t-designs: $t$-wise independence in the quantum world. In Proceedings of the 22nd Annual IEEE conference on Computational Complexity, pages 129-140, 2007.

[BB84] C. Bennett and G. Brassard. Quantum cryptography: Public key distribution and coin tossing. In Proceedings of the IEEE International Conference on Computers, Systems, and Signal Processing, pages 175-179, 1984.

[BCDM00] D. Bruß, M. Cinchetti, G. D'Ariano, and C. Macchiavello. Phase covariant quantum cloning. Physical Review A, 62:012302, 2000.

[BH96] V. Bužek and M. Hillery. Quantum copying: Beyond the no-cloning theorem. Physical Review A, 54(3):1844-1852, 1996.

[BV04] S. Boyd and L. Vandenberghe. Convex Optimization. Cambridge University Press, 2004.

[CF06] N. Cerf and J. Fiurášek. Optical quantum cloning, volume 49 of Progress in Optics, chapter 6, pages 455-545. Elsevier, 2006.

[Cho75] M.-D. Choi. Completely positive linear maps on complex matrices. Linear Algebra and Its Applications, 10(3):285-290, 1975.

[dK02] E. de Klerk. Aspects of Semidefinite Programming - Interior Point Algorithms and Selected Applications, volume 65 of Applied Optimization. Kluwer Academic Publishers, Dordrecht, 2002.

[EMV03] Y. Eldar, A. Megretski, and G. Verghese. Designing optimal quantum detectors via semidefinite programming. IEEE Transactions on Information Theory, 49(4):1007-1012, 2003.

${ }^{11}$ http://theoreticalphysics.stackexchange.com/questions/370/ 
[EW01] T. Eggeling and R. Werner. Separability properties of tripartite states with $U \otimes U \otimes U$ symmetry. Physical Review A, 63(4):042111, 2001.

$\left[\mathrm{FGH}^{+} 10\right]$ E. Farhi, D. Gosset, A. Hassidim, A. Lutomirski, and P. Shor. Quantum money from knots. Available as arXiv.org e-Print 1004.5127, 2010.

[Gav11] D. Gavinsky. Quantum money with classical verification. Available as arXiv.org ePrint 1109.0372, 2011.

[Got02] D. Gottesman. Uncloneable encryption. Available as arXiv.org e-Print quantph/0210062, 2002.

[GW07] G. Gutoski and J. Watrous. Toward a general theory of quantum games. In Proceedings of the 39th Annual ACM Symposium on Theory of Computing, pages 565-574, 2007.

[Jam72] A. Jamiołkowski. Linear transformations which preserve trace and positive semidefiniteness of operators. Reports on Mathematical Physics, 3(4):275-278, 1972.

$\left[\mathrm{LAF}^{+} 10\right]$ A. Lutomirski, S. Aaronson, E. Farhi, D. Gosset, A. Hassidim, J. Kelner, and P. Shor. Breaking and making quantum money: toward a new quantum cryptographic protocol. In Proceedings of Innovations in Computer Science (ICS), pages 20-31, 2010.

[Lov03] L. Lovász. Semidefinite programs and combinatorial optimization. Recent Advances in Algorithms and Combinatorics, 2003.

[LSP98] H. Lo, T. Spiller, and S. Popescu. Introduction to Quantum Computation and Information. World Scientific Publishing Company, 1998.

[Lut10] A. Lutomirski. An online attack against Wiesner's quantum money. Available as arXiv.org e-Print 1010.0256, 2010.

[May01] D. Mayers. Unconditional security in quantum cryptography. Journal of the ACM, 48:351-406, 2001.

[MS07] R. Mittal and M. Szegedy. Product rules in semidefinite programming. In Fundamentals of Computation Theory, volume 4639 of Lecture Notes in Computer Science, pages 435-445. Springer-Verlag, 2007.

[MW11] A. Molina and J. Watrous. Hedging bets with correlated quantum strategies. Available as arXiv.org e-Print 1104.1140, 2011.

[NC00] M. A. Nielsen and I. L. Chuang. Quantum Computation and Quantum Information. Cambridge University Press, 2000.

$\left[\mathrm{PYJ}^{+} 11\right] \quad$ F. Pastawski, N. Y. Yao, L. Jiang, M. D. Lukin, and J. I. Cirac. Unforgeable noisetolerant quantum tokens. Available as arXiv.org e-Print 1112.5456, 2011.

[RBKSC04] J. Renes, R. Blume-Kohout, A. Scott, and C. Caves. Symmetric informationally complete quantum measurements. Journal of Mathematical Physics, 45:2171-2180, 2004.

[SP00] P. Shor and J. Preskill. Simple proof of security of the BB84 quantum key distribution protocol. Physical Review Letters, 85(2):441-444, 2000. 
[TR11] M. Tomamichel and R. Renner. Uncertainty relation for smooth entropies. Physical Review Letters, 106:110506, 2011.

[VB96] L. Vandenberghe and S. Boyd. Semidefinite programming. SIAM Review, 38(1):49-95, 1996.

[VW11] T. Vidick and S. Wehner. Does ignorance of the whole imply ignorance of the parts? Large violations of noncontextuality in quantum theory. Physical Review Letters, 107:030402, 2011.

[Wat11] J. Watrous. Lecture notes on Theory of Quantum Information. Available at http://www.cs.uwaterloo.ca/ watrous/CS766/, 2011.

[Wer98] R. Werner. Optimal cloning of pure states. Physical Review A, 58:1827-1832, 1998.

[Wie83] S. Wiesner. Conjugate coding. SIGACT News, 15(1):78-88, 1983.

[WZ82] W. Wootters and W. Zurek. A single quantum state cannot be cloned. Nature, 299:802803, 1982. 